\title{
Genomic homogeneity in fibrolamellar carcinomas
}

\author{
Y Sirivatanauksorn, V Sirivatanauksorn, N R Lemoine, R C N Williamson, B R Davidson
}

\begin{abstract}
Background-Fibrolamellar carcinoma (FLC) is a variant of hepatocellular carcinoma (HCC) with distinctive clinical and histological features. To date there have been few studies on the genotypic aspects of FLC and no previous attempts have been made to use the arbitrarily primedpolymerase chain reaction (AP-PCR) technique to detect genetic alterations in this disease.

Aim-The aim of this study was to assess the degree of genomic heterogeneity of FLC using the AP-PCR technique.

Methods-A total of 50 tissue samples of primary and metastatic FLCs from seven patients were microdissected. AP-PCR amplification of each genomic DNA sample was carried out using two arbitrary primers.

Results-DNA fingerprints of the primary FLCs and all their metastatic lesions (both synchronous and metachronous disease) were identical in an individual patient. The fingerprints were different between tumours of different patients. No evidence of intratumour heterogeneity was observed.

Conclusions-Such genomic homogeneity in FLCs may explain their indolent growth. The absence of clonal evolution, which is present in other tumours (particularly HCCs), may explain the distinct behaviour in this tumour. The tumorigenic pathway and degree of somatic genomic changes in this disease may be less complex than in HCC. (Gut 2001;49:82-86)
\end{abstract}

Keywords: fibrolamellar carcinoma; hepatocellular carcinoma; DNA fingerprint; arbitrarily primed-polymerase chain reaction; laser capture microdissection

Research Fund

Molecular Oncology

Unit, Imperial College

School of Medicine,

Hammersmith

Hospital, London, UK

V Sirivatanauksorn

N R Lemoine

Department of Gastrointestinal Surgery, Imperial College School of Medicine,

Hammersmith

Hospital, London, UK

R C N Williamson

Correspondence to: Professor BR Davidson. b.davidson@rfc.ucl.ac.uk

Accepted for publication 4 September 2000

Fibrolamellar carcinoma (FLC) is an uncommon variant of hepatocellular carcinoma (HCC) which has a particular type of histological appearance and clinical behaviour. It constitutes approximately $7 \%$ of primary liver cancers $^{1}$ and is relatively common among Whites, occurring with equal frequency in men and women. The clinical symptoms are usually non-specific and often comprise discomfort related to the presence of a large hepatic mass. The tumour typically arises in non-cirrhotic liver and has serum biochemical markers that differ from the usual HCC. Alpha fetoprotein is usually normal in $\mathrm{FLC}^{2}$ but levels of serum vitamin $\mathrm{B} 12$ binding globulin ${ }^{3}$ and neurotensin ${ }^{4}$ are often increased. Histologically, FLC is characterised by large polygonal shaped cells with eosinophilic granular cytoplasm separated into nests and sheets by lamellar bands of collagen. The nuclei are vesicular and contain prominent eosinophilic nucleoli. Pleomorphism and mitoses are minimal. ${ }^{1}$

Surgical resection is the treatment of choice for this tumour. Complete surgical resection results in a cure in $50-75 \%$ of patients. ${ }^{5}$ Extensive surgical resection is warranted in FLC even with very large tumours. ${ }^{6}$ In a large study of 41 patients with FLC, long term survival was frequently achieved with radical surgical treatment; cumulative survival rates at $1,3,5$, and 10 years were $98 \%, 72 \%, 66 \%$, and $47 \%$, respectively. ${ }^{7}$ Surgery may even be beneficial with recurrent and metastatic tumour either with or without chemotherapy. ${ }^{6}$

Recently, HCC has been shown to contain subpopulations of heterogeneous cellular differentiation within each tumour which can be demonstrated using the arbitrarily primedpolymerase chain reaction (AP-PCR) technique. ${ }^{89}$ This is one of the novel fingerprinting techniques described by Welsh and McClelland $(1990)^{10}$ and is widely used to amplify sections of DNA/RNA for identifying gene structure or matching tissue specimens. ${ }^{11-13}$ AP-PCR allows the detection of polymorphisms without prior knowledge of nucleotide sequence and is based on the selective amplification of genomic sequences that, by chance, are flanked by adequate matches to an arbitrarily chosen primer. Despite the obvious clinical differences in the natural history of FLC in comparison with HCC, to date there have been few studies on the genotypic aspects of FLC and no previous attempts have been made to use AP-PCR to investigate evidence of genetic alterations in this disease. In this study, the degree of genomic heterogeneity of FLC was assessed using the AP-PCR technique.

\section{Materials and methods}

Tissue samples used in this study were formalin fixed, paraffin embedded tissues of primary and metastatic FLC lesions from seven patients undergoing elective surgical resection. Details of tissue samples are summarised in table 1 .

NEEDLE MICRODISSECTION AND DNA PREPARATION (PATIENTS NOS 1-3)

One $5 \mu \mathrm{m}$ section and $10 \mu \mathrm{m}$ sections were serially cut from each archival block onto glass slides by microtome. The first $5 \mu \mathrm{m}$ section was

Abbreviations used in this paper: FLC, fibrolamellar carcinoma; HCC, hepatocellular carcinoma; AP-PCR, arbitrarily primed-polymerase chain reaction; LCM, laser capture microdissection. 
stained with haematoxylin and eosin for histological confirmation. The remaining $10 \mu \mathrm{m}$ sections were stained with $0.1 \%$ toluidine blue solution for 10 minutes. Tissue samples were separately prepared by microdissection using sterile 27 gauge needles with the assistance of a dissection microscope and referenced to a section stained with haematoxylin and eosin. Deparaffinisation was achieved by incubating in xylene for 20 minutes at $55^{\circ} \mathrm{C}$ and then washing twice with absolute ethanol. DNA was prepared by incubating tissue in lysis buffer ( 10 $\mathrm{mM}$ Tris $\mathrm{Cl}, \mathrm{pH} 8.0,50 \mathrm{mM} \mathrm{KCl}, 2.5 \mathrm{mM}$ $\mathrm{MgCl}_{2}, 0.1 \mathrm{mg} / \mathrm{ml}$ gelatin, $0.45 \% \mathrm{NP}-40$, $0.45 \%$ Tween 20 , proteinase $\mathrm{K} 500 \mu \mathrm{g} / \mathrm{ml}$ ) overnight at $55^{\circ} \mathrm{C}$. The lysate was subjected to three phenol/chloroform/isoamyl alcohol (25:24:1, by volume) extractions. After ethanol precipitating, washing, and drying the DNA, it was resuspended in sterile water.

LASER CAPTURE MICRODISSECTION (LCM) AND DNA PREPARATION (PATIENTS NOS 4-7)

Two $10 \mu \mathrm{m}$ sections were serially cut from each archival block onto glass slides by microtome and stained with haematoxylin and eosin. The first section was for histological confirmation. The tissues were separately microdissected by laser capture microdissection (LCM) (Arcturus Engineering Inc., California, USA). The system employs an ethylene vinyl acetate polymer layer coated onto the underside of a rigid flat vial cap. Under the microscope, the cells of choice can be viewed through the film, and the pulsed laser beam activates a precise spot on the transfer film. At this precise location, the film immediately above the cells of interest melts and fuses with the underlying cells. The cap is lifted off the tissue and placed directly onto a $0.5 \mathrm{ml}$ microfuge tube containing $50 \mu \mathrm{l}$ of proteinase $\mathrm{K}$ buffer. The tube was inverted and incubated overnight at $37^{\circ} \mathrm{C}$. After the incubation period, the tube was centrifuged at $13000 \mathrm{rpm}$ for five minutes and the cap was removed. Then the buffer was inactivated at $95^{\circ} \mathrm{C}$ for 10 minutes and the solution was ready to use as a template for PCR. Two sectors of each section were microdissected separately.

The DNA samples obtained by the microdissection techniques were qualitatively assessed; $4 \mu \mathrm{l}$ of DNA solution were used for amplification with GAPDH primers in a total volume of $50 \mu \mathrm{l}$ containing $50 \mathrm{mM} \mathrm{KCl}, 10 \mathrm{mM}$ Tris $\mathrm{Cl}$, $\mathrm{pH} 8.3,1.5 \mathrm{mM} \mathrm{MgCl}, 100 \mathrm{pM}$ primers, and 5 units of Taq polymerase (Bioline, London, UK). Templates were denatured for five minutes at $95^{\circ} \mathrm{C}$ and subjected to 35 cycles at $94^{\circ} \mathrm{C}$ for one minute, $55^{\circ} \mathrm{C}$ for one minute, and $72^{\circ} \mathrm{C}$ for two minutes. The PCR products were run on $1.5 \%$ agarose gel staining with ethidium bromide (data not shown). Fingerprinting of DNA samples was performed using the APPCR technique.

AP-PCR AMPLIFICATION

Two different arbitrary primers (AR3: 5'GCGAATTCATGTACGTCAGG-3 ${ }^{\prime 14}$ and ZF3: $\quad$ '-CCCCACCGGAGAGAAACC- $3^{15}$ ) were used to amplify the genomic DNA of each sample. These primers have previously been shown to provide useful information on the genotypic profile of HCC. ${ }^{89}$ An arbitrary primer $(5 \mu \mathrm{M})$ was labelled with $\gamma-{ }^{33} \mathrm{P}$ (Amersham Pharmacia Biotech, Buckinghamshire, UK) using T4 polynucleotide kinase (Bioline, London, UK) at $37^{\circ} \mathrm{C}$ for one hour. AP-PCR amplifications were carried out using $200 \mathrm{ng}$ of template DNA in a reaction mixture containing $5 \mu \mathrm{M}{ }^{33} \mathrm{P}$ labelled arbitrary primer, $0.2 \mathrm{mM}$ each dNTP (Bioline, London, UK), $10 \mathrm{mM}$ Tris Cl, pH 9.2, $3.5 \mathrm{mM} \mathrm{MgCl}_{2}, 75 \mathrm{mM} \mathrm{KCl,}$ and 0.5 units of Taq DNA polymerase (Bioline, London, UK) in a final volume of $25 \mu \mathrm{l}$. The reactions were overlaid with mineral oil and cycled through the following temperature profile: $94^{\circ} \mathrm{C}$ for one minute to denature, $45^{\circ} \mathrm{C}$ for
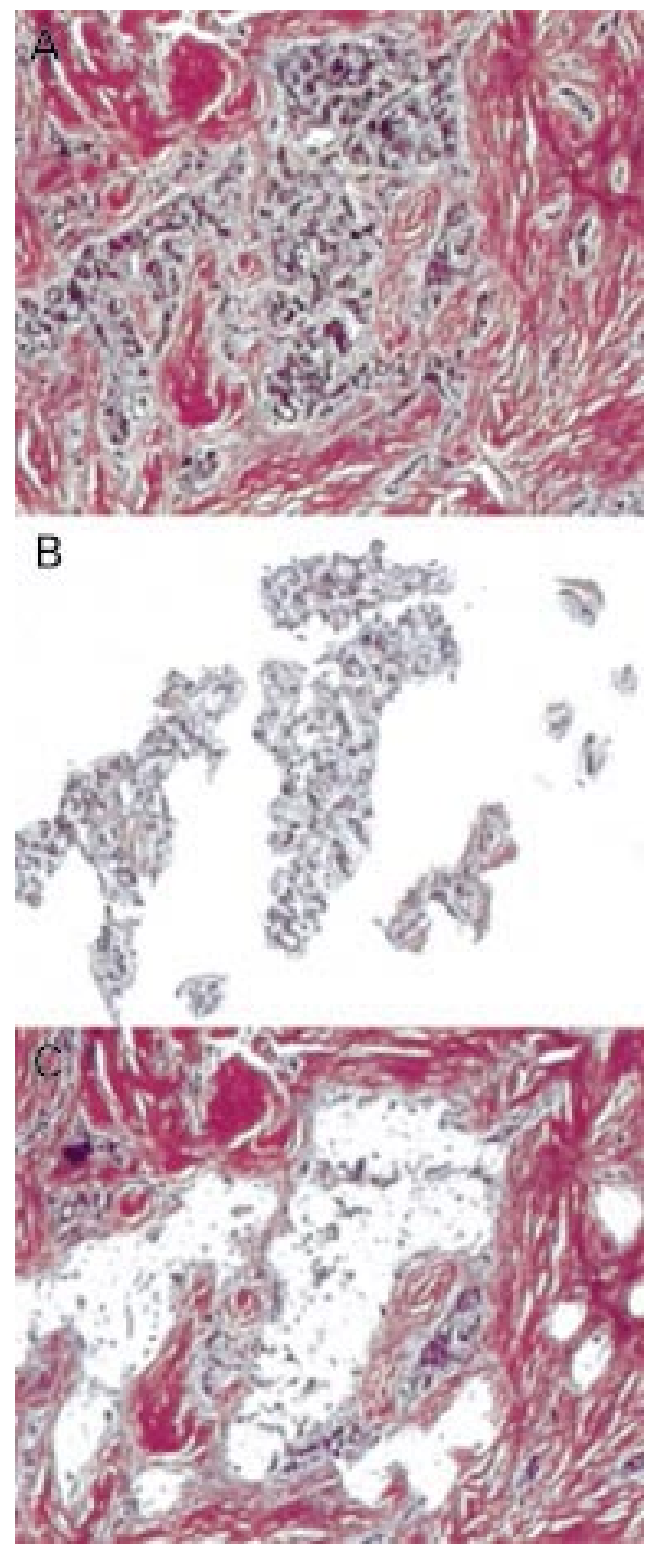

Figure 1 Laser capture microdissection (LCM) of a fibrolamellar carcinoma (FLC) nodule. As no coverslip is used in LCM, the reduction in refractive index means that most light passing through the tissue is scattered which can obscure cellular detail at high magnifications. (A) A $10 \mu \mathrm{m}$ thick paraffin embedded section of FLC tissue stained with haematoxylin and eosin. The tumour cells are separated into nests and sheets by dense lamellar bands of collagen. (B) Selected tumour cells transferred to the film of the vial caps. (C) The residual tissue section after laser capture. 
Table 1 List of fibrolamellar carcinoma (FLC) samples

\begin{tabular}{|c|c|c|c|}
\hline $\begin{array}{l}\text { Patient } \\
\text { No }\end{array}$ & Primary & Metastatic sites & $\begin{array}{l}\text { No of } \\
\text { samples }\end{array}$ \\
\hline 1 & FLC & $\begin{array}{l}\text { Left ovarian mass, appendiceal nodule, lymph nodes } \\
\text { (infrapancreatic, inferior vena cava), lesser omentum nodules, } \\
\text { peritoneum }\end{array}$ & 10 \\
\hline 2 & FLC & Lungs & 6 \\
\hline 3 & FLC & Intrahepatic recurrent nodules & 3 \\
\hline 4 & FLC & $\begin{array}{l}\text { Intrahepatic satellite nodules }{ }^{\star} \text {, lymph nodes (retropancreatic }{ }^{\star} \text {, } \\
\text { para-aortic }{ }^{\star} \text {, preaortic, infrapancreatic) }\end{array}$ & 12 \\
\hline 5 & FLC & Lungs, left portal nodule & 9 \\
\hline 6 & FLC & Retroduodenal lymph nodes* & 3 \\
\hline 7 & FLC & $\begin{array}{l}\text { Lymph nodes }{ }^{\star} \text { (pancreatic, hepatic artery, gastric, mesenteric), } \\
\text { small bowel }^{\star} \text {, chest wall }{ }^{\star}\end{array}$ & 7 \\
\hline
\end{tabular}

${ }^{\star}$ Synchronous tumours.
Whatman $3 \mathrm{MM}$ paper, dried under vacuum, and exposed to $x$ ray film (BioMax MR, Kodak, USA) for 24-48 hours at room temperature.

\section{Results}

Comparison between the needle based and LCM techniques have been previously described. ${ }^{9}$ Although manual microdissection (patients Nos 1-3) was time consuming, labour intensive, and required a high degree of manual skill, it was successfully used to isolate tumour cells from sheets of collagen. The LCM technique (patients Nos 4-7) was simple, fast, required no moving parts, involved no manual microdissection or manipulations, and enabled one step transfers. Moreover, the manually microdissected cells can detach from the tip of the needle during transfer whereas cells obtained by LCM adhere to the film where they retain their morphological features and the operator can verify that the correct cells have been procured (fig 1). Tumour cells are large and polygonal. Islands of tumour are separated by dense collagenous fibrous tissue.

A total of 50 samples of primary and metastatic FLCs from seven patients were analysed (table 1). Each tumour was microdissected and tissue was taken from two sectors of each tumour (for assessment of intratumoral (Life Technol phoresis, the gel was transferred to a piece of
B

P Ov Ap LNP LNIVC LO1 LO2 Peri $\begin{array}{llllllllllllllll}1 & 2 & 1 & 2 & 1 & 2 & 1 & 2 & 1 & 2 & 1 & 2 & 1 & 2 & 1 & 2\end{array}$

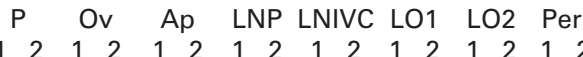
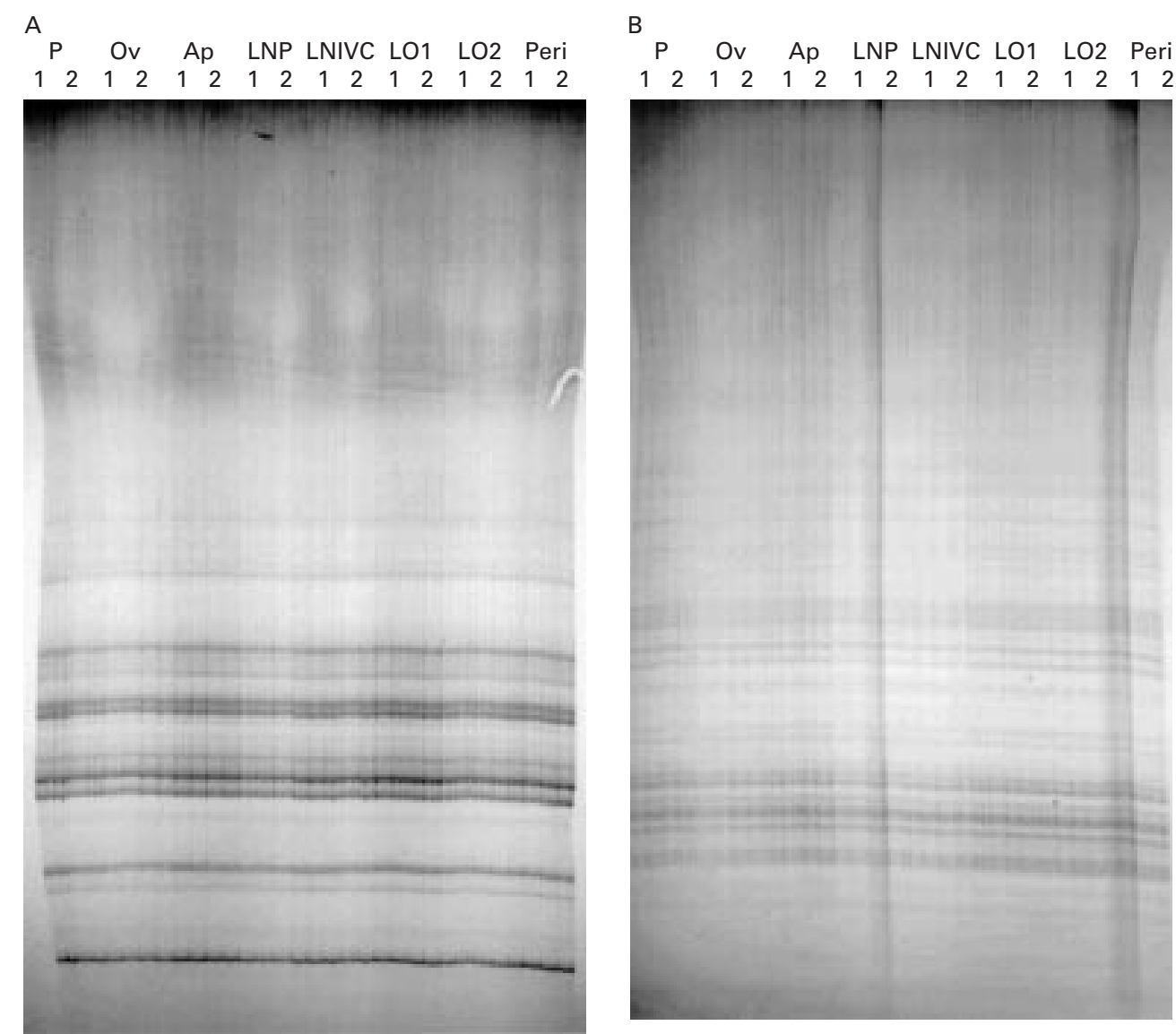

Figure 2 Arbitrarily primed-polymerase chain reaction (AP-PCR) analysis of primary fibrolamellar carcinomas (FLC) and their metastatic lesions from patient No 1. Autoradiograms of polyacrylamide gel electrophoresis of ${ }^{33} P$ labelled DNA fragments amplified by AP-PCR. Genomic DNA from each sample was amplified with the AR3 (A) and ZF3 (B) primers. $P$, primary FLC; Ov, left ovarian mass; Ap, appendiceal nodule; LNP, infrapancreatic lymph nodes; LNIVC, inferior vena cava lymph nodes; LO1/LO2, lesser omentum nodules; Peri, peritoneum; (number of sectors is represented at the top). 
A

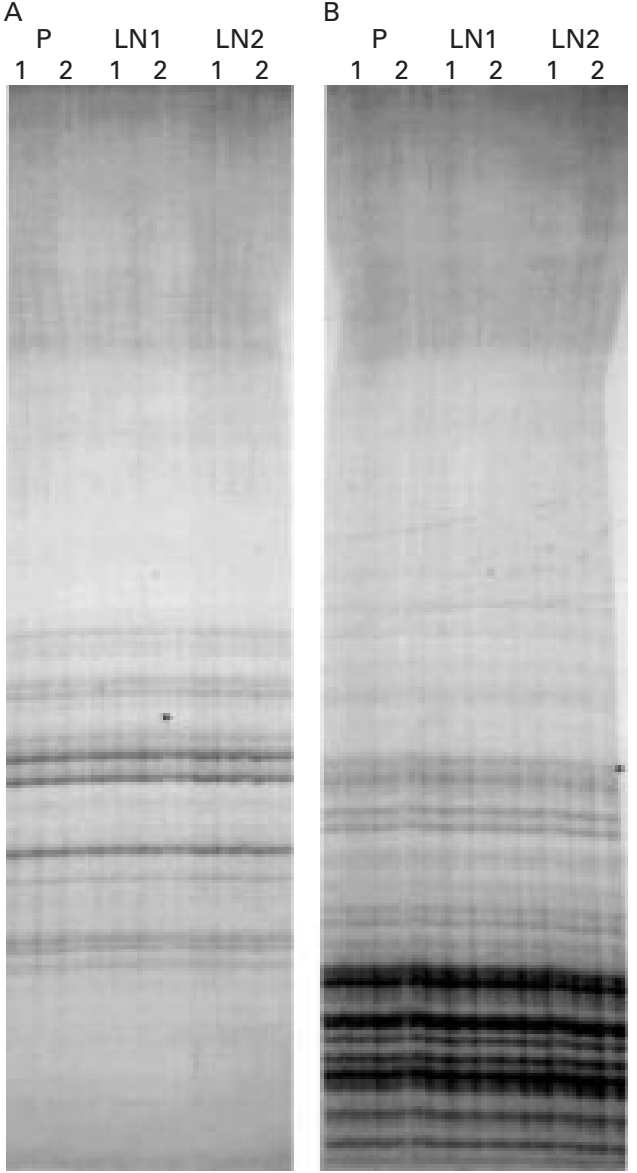

Figure 3 Arbitrarily primed-polymerase chain reaction (AP-PCR) analysis of primary fibrolamellar carcinomas (FLC) and their metastatic lesions from patient No 6. Autoradiograms of polyacrylamide gel electrophoresis of ${ }^{33} \mathrm{P}$ labelled DNA fragments amplified by AP-PCR. Genomic $D N A$ from each sample was amplified with the AR3 $(A)$ and ZF3 (B) primers. P, primary FLC; $L N$, lymph nodes metastases; (number of sectors is represented at the top).

genotypic variation). Using AP-PCR with each of the two arbitrary primers, we generated specific and reproducible DNA fingerprints. Each PCR reaction was performed in duplicate with equal aliquots of sample template DNA, and the fingerprints of these paired reactions were identical. DNA fingerprints of the primary FLCs and all their metastatic lesions (both synchronous and metachronous diseases) were identical in the same patient (figs 2,3 ). The fingerprints were different between tumours from different patients. The AP-PCR results were the same when compared between different sectors of the same tumour mass, independent of whether these were primary or metastatic lesions. No evidence of intratumour heterogeneity was observed.

\section{Discussion}

Laser assisted microdissection assists in the molecular study of FLCs because these cancers contain dense fibrous stromal components which may complicate analysis of a particular tumour cell population. The identity of the transferred cells attached to the film can be viewed and recorded by image capture. It is obviously an advantage to use microdissected cell samples in molecular analysis because the confounding effect of contaminating cells is eliminated. An increase in sensitivity of more than $50 \%$ in allelic imbalance analysis has been reported using microdissected cell populations compared with crushed tumour samples. ${ }^{16}$ Precise microdissection of phenotypically similar prostatic cancer tissue samples and analysis of the DNA for loss of heterozygosity revealed intratumoral genetic heterogeneity and provided evidence for the multifocality of tumour development in the prostate. ${ }^{17}$ AP-PCR is a highly specific technique that can generate species specific and individual diagnostic DNA fingerprints. ${ }^{18}$ This method has been applied to detect and isolate novel DNA fragments associated with somatic genomic changes in colorectal cancer, lung cancer, and astrocytoma. ${ }^{11}{ }^{1920}$ Moreover, it has been shown to be an uncomplicated and effective approach for scanning the genomes of tumour samples to show the evolution of differences. ${ }^{9}$

FLC has distinctive histological and clinical features. It occurs predominantly in children and young adults without a predilection for either sex, and it carries a better prognosis than the more common HCC. The indolent growth of FLC is especially obvious when recurrences develop. Even after the diagnosis of tumour recurrence, survival at one, three, and five years has been reported to be $75 \%, 48 \%$, and $28 \%$ respectively. ${ }^{7}$ Several studies have attempted to associate the differences in natural history of this cancer from those of HCC. Flow cytometric analysis suggested that the DNA content in FLC is not directly related to clinical behaviour. ${ }^{21}{ }^{22}$ In contrast, the overall survival rate of HCC patients with DNA aneuploid tumours was significantly poorer than that of patients with diploid tumours. ${ }^{23}$ Positive staining for neurone specific enolase, the finding of neurosecretory type granules at the ultrastructural level, and raised serum levels of neurotensin suggest neuroendocrine differentiation of this disease. ${ }^{424}$ Neurotensin, an important regulatory peptide in the gut that facilitates translocation of fatty acids from the intestinal lumen, is a useful marker to differentiate FLC from other tumours. ${ }^{25}$ Neurotensin is expressed in fetal human liver and in FLC, but not in the adult liver, focal nodular hyperplasia, or in HCC. ${ }^{25} 26$ However, the absence of neurotensin receptor expression emphasises the fact that neurotensin probably does not play a primary role in liver growth.

This is the first report of genomic homogeneity in FLC and metastatic lesions. Absence of the clonal evolution that is found in other tumours, particularly HCC, ${ }^{9}$ may explain the distinct behaviour of FLC. Patients with this tumour differ from those with HCC in terms of age at presentation, absence of underlying liver disease, and tumour markers. These characteristics may reflect different aetiological factors because there is no recognised association of FLC with viral hepatitis, alcohol, cirrhosis, oral contraceptives, or genetic disorders such as Wilson's disease. Tumorigenesis and degree of somatic genomic changes (rearrangements, losses, or gains) in this disease may be less complex than in HCC. None of nine patients 
with FLCs had p53 mutations (exons 5-8) analysed by denaturing gradient gel electrophoresis, single strand conformation polymorphism, and gene sequencing. ${ }^{27}$ Moreover, in a loss of heterozygosity study using restriction fragment length polymorphism probes for various chromosomes, the rate of allelic loss in FLC was infrequent $(3.6 \%)$ compared with that in HCC $(16.1 \%){ }^{28}{ }^{29}$ The cell of origin of FLC remains uncertain. Whether FLC and HCC represent extremes of the spectrum of one disease entity remains uncertain. Interestingly, there are few reports of the synchronous development of FLC and ordinary HCC. ${ }^{30}{ }^{31}$ There is no defined at risk group to screen other than the young, and thus there is little prospect of improving the prognosis in this way.

1 Craig JR, Peters RL, Edmondson HA, et al. Fibrolamellar carcinoma of the liver: a tumor of adolescents and young adults with distinctive clinico-pathologic features. Cancer 1980;4:372-9

2 Berman MA, Burnham JA, Sheahan DG. Fibrolamellar carcinoma of the liver: an immunohistochemical study of nineteen cases and a review of the literature. Hum Pathol 1988;19:784-94

3 Paradinas FJ, Melia WM, Wilkinson ML, et al. High serum vitamin B12 binding capacity as a marker of the fibrolamellar variant of hepatocellular carcinoma. $B M^{\mathcal{F}}$ 1982;285:840-2.

4 Collier NA, Weinbren K, Bloom SR, et al. Neurotensin secretion by fibrolamellar carcinoma of the liver. Lancet 1984;1:538-40.

5 Starzl TE, Iwatuski S, Shaw BW Jr, et al. Treatment of fibrolamellar hepatoma with partial or total hepatectomy and lamellar hepatoma with partial or total hepatectomy and transplant.

6 Soreide O, Czerniak A, Bradpiece $\mathrm{H}$, et al. Characteristics of fibrolamellar hepatocellular carcinoma. A study of nine cases and a review of the literature. Am f Surg 1986;151:518-23.

7 Pinna AD, Iwatsuki S, Lee RG, et al. Treatment of fibrolamellar hepatoma with subtotal hepatectomy or transplantation. Hepatology 1997;26:877-83.

8 Sirivatanauksorn Y, Sirivatanauksorn V, Bhattacharya S, et al. Genomic heterogeneity in synchronous hepatocellular carcinomas. Gut 1999;45:761-5.

9 Sirivatanauksorn Y, Sirivatanauksorn V, Bhattacharya S, et al. Evolution of genetic abnormalities in hepatocellular carcinomas demonstrated by DNA fingerprinting. I Patho 1999;189:344-50.

10 Welsh J, McClelland M. Fingerprinting genomes using PCR with arbitrary primers. Nucleic Acids Res 1990;18:7213-8.

11 Peinado MA, Malkhosyan S, Velazquez A, et al. Isolation and characterisation of allelic losses and gains in colorectal tumors by arbitrarily primed polymerase
Proc Natl Acad Sci USA 1992;89:10065-9.

12 Welsh J, Pretzman C, Postic D, et al. Genomic fingerprinting by arbitrarily primed polymerase chain reaction resolves
Borrelia burgdorferi into three distinct phyletic groups. Int Syst Bacteriol 1992;42:370-3.

13 Innov Y, Peinado MA, Malkhosyan S, et al. Ubiquitous somatic mutations in simple repeated sequences reveal a new mechanism for colonic carcinogenesis. Nature 1993; 363:558-61.

14 Achille A, Biasi MO, Zamboni G, et al. Chromosome 7q allelic losses in pancreatic carcinoma. Cancer Res 1996;56: 3808-13.

15 Welsh J, McClelland M. Genomic fingerprints produced by PCR with concensus tRNA gene primers. Nucleic Acids Res 1991;19:861-6.

16 Giercksky HE, Thorstensen L, Qvist H, et al. Comparison of genetic changes in frozen biopsies and microdissected archival material from the same colorectal liver metastases. Diagn Mol Pathol 1997;6:318-25.

17 Macintosh CA, Stower M, Reid N, et al. Precise microdissection of human prostate cancers reveals genotypic heterogeneity. Cancer Res 1998;58:23-8.

18 Schlegel J, Vogt T, Munkel K, et al. DNA fingerprinting of mammalian cell lines using nonradioactive arbitrarily primed PCR (AP-PCR). Biotechniques 1996;20:78-80

19 Kohno T, Morishita K, Takano H, et al. Homozygous deletion at chromosome $2 \mathrm{q} 33$ in human small-cell lung carcinoma identified by arbitrarily primed PCR genomic fingerprinting. Oncogene 1994;9:103-8.

20 Saitoh Y, Bruner JM, Levin VA, et al. Identification of allelic loss on chromosome arm $6 \mathrm{p}$ in human astrocytomas by arbitrarily primed polymerase chain reaction. Genes Chromosomes Cancer 1998;22:165-70.

21 Saul SH, Titelbaum DS, Gansler TS, et al. The fibrolamellar variant of hepatocellular carcinoma: its association with focal nodular hyperplasia. Cancer 1987;60:3049-55.

22 Orsatti G, Greenberg PD, Rolfes DB, et al. DNA ploidy of fibrolamellar hepatocellular carcinoma by image analysis. Hum Pathol 1994;25:936-9.

23 Mise K, Tashiro S, Yogita S, et al. Assessment of the biological malignancy of hepatocellular carcinoma: relationship to clinicopathological factors and prognosis. Clin Cancer Res 1998;4:1475-82.

24 Payne CM, Nagle RB, Paplanus SH, et al. Fibrolamellar carcinoma of liver. A primary malignant oncocytic carcinoid? Ultrastruct Pathol 1986;10:539-52.

25 Ehrenfried JA, Zhou Z, Thompson JC, et al. Expression of the neurotensin gene in fetal human liver and fibrolamellar carcinoma. Ann Surg 1994;220:484-9.

26 Reubi JC, Waser B, Schaer JC, et al. Neurotensin receptors in human neoplasms: high incidence in Ewing's sarcomas. Int F Cancer 1999;82:213-8.

27 Honda K, Sbisa E, Tullo A, et al. p53 mutation is a poor prognostic indicator for survival in patients with hepatocellular carcinoma undergoing surgical tumour ablation. $\mathrm{Br} 7$ Cancer 1998;77:776-82

28 Ding SF, Delhanty JDA, Bowles L, et al. Infrequent chromosome allele loss in fibrolamellar carcinoma. Br $\mathrm{f}$ Cancer 1993;67:244-6.

29 Ding SF, Habib NA, Dooley J, et al. Loss of constitutional heterozygosity on chromosome $5 \mathrm{q}$ in hepatocellular carcinoma without cirrhosis. Br f Cancer 1991;64:1083-7.

30 Reuland P, Aicher KP, Brambs HJ, et al. Incidental finding of a hepatic lesion: Differential diagnostic problems for fibrolamellar hepatic carcinoma. F Nucl Med 1994;35: 1342-6.

31 Okano A, Hajiro K, Takakuwa H, et al. Fibrolamellar carcinoma of the liver with a mixture of ordinary hepatocellular carcinoma: A case report. Am f Gastroenterol 1998;93: $1144-5$. 